\title{
Développer des compétences d'expert dans le domaine des écosystèmes littoraux
}

\section{Christian Reynaud et Daniel Favre}

\section{(2) OpenEdition \\ 1 Journals}

Édition électronique

URL : http://journals.openedition.org/trema/2073

DOI : 10.4000/trema.2073

ISSN : 2107-0997

Éditeur

Faculté d'Éducation de l'université de Montpellier

\section{Édition imprimée}

Date de publication : 1 septembre 1996

Pagination : 139-154

ISSN : 1167-315X

\section{Référence électronique}

Christian Reynaud et Daniel Favre, « Développer des compétences d'expert dans le domaine des écosystèmes littoraux », Tréma [En ligne], 9-10 | 1996, mis en ligne le 18 septembre 2013, consulté le 19 avril 2019. URL : http://journals.openedition.org/trema/2073 ; DOI : 10.4000/trema.2073

Ce document a été généré automatiquement le 19 avril 2019.

Trema 


\title{
Développer des compétences d'expert dans le domaine des écosystèmes littoraux
}

\author{
Christian Reynaud et Daniel Favre
}

\section{Introduction}

1 Considéré sous l'angle de son histoire, l'écologie scientifique s'est progressivement dissociée de l'étude naturaliste, fondée sur l'observation des êtres vivants, en développant une écologie appliquée dans laquelle se confrontent la demande sociale et les problématiques théoriques et dont les négociations internationales, qu'il s'agisse de la régulation de l'effet de serre, de la préservation de la biodiversité ou de la protection de la couche d'ozone, sont les manifestations ostentatoires (Deléage, 1991 ; Drouin, 1991). De fait, les chercheurs en écologie ne sont pas toujours préoccupés de recherche fondamentale : ce sont aussi, souvent, des experts sollicités pour résoudre des problèmes pratiques. De cette confrontation peuvent naître des concepts écologiques, comme en témoigne l'œuvre de Möbius basée sur une analyse économique de l'exploitation des huîtres et qui va consacrer le concept de « biocénose » (Ibid.).

De manière semblable, depuis le début des années 80, Guelorget et Peithuisot ont introduit le "domaine paralique $»^{1}$ en géologie et en écologie des milieux littoraux pour regrouper un ensemble de milieux, que leurs travaux rendaient comparables (Guelorget et Perthuisot, 1983). Afin de favoriser la diffusion du " concept d'écosystèmes paraliques » nous avons été amenés à collaborer avec les chercheurs concernés par l'étude de ces milieux et nous travaillons actuellement sur une formalisation susceptible de faciliter sa communication et son appropriation.

Dans cette optique, une définition du concept a été élaborée à partir des attributs du concept. Selon Barth, les attributs essentiels ${ }^{2}$ sont les qualités qui permettent de spécifier un concept, de distinguer une catégorie d'objets (Barth, 1993). Appliqués aux écosystèmes 
paraliques, six attributs permettent de différencier ces écosystèmes des autres types de milieux (Reynaud et al., 1995a).

4 L'efficacité du modèle ainsi défini pour des activités de communication a alors été testée dans le cadre didactique stricto sensu. Dans ce cadre, l'objectif de la communication est explicitement l'acquisition d'un savoir par les apprenants. Mais, si " "savoir", c'est d'abord être capable d'utiliser ce qu'on a appris, de le mobiliser pour résoudre un problème ou clarifier une situation " (Giordan et De Vecchi, 1987, p. 5), l'enseignement ne peut pas transmettre un savoir en soi, il doit trouver un moyen de le présenter comme un système producteur de sens et efficace dans la pratique de tout un chacun. Comme Brousseau l'a montré pour l'apprentissage des mathématiques, nous pensons que c'est à travers des situations et des problèmes à résoudre qu'un concept acquiert du sens pour un individu (Brousseau, 1986). L'apprentissage par résolution de problème (Problem Based Learning) semble alors donner des résultats attrayants (Pochet, 1995).

5 Pour transposer ce type d'approche dans notre problématique, nous avons élaboré des «situations-problèmes » à partir de rapports d'expertise. Ces énoncés comportent les informations nécessaires pour reproduire la démarche de l'expert, en la limitant au champ d'application du concept d'écosystème paralique. Ces situations-problèmes constituent, avec le modèle formalisé à partir des attributs du concept, les supports pratiques utilisés dans le dispositif qui va maintenant être décrit.

\section{Présentation de la séance}

La séance est prévue pour une durée approximative de trois heures. Elle est présentée comme une séquence d'application d'un concept récent d'écologie marine pour lequel on essaye de montrer l'intérêt d'une approche centrée sur celui-ci. L'utilisation de situations-problèmes - issues d'études d'expertise sur ces milieux et pour lesquelles il est nécessaire de mobiliser et de maîtriser le concept - est mentionnée comme accompagnement de la démarche. Les documents proposés en cours de séance sont des supports de travail qui sont récupérés à la fin (ils sont présentés dans la suite de ce texte).

7 Le concept, objet d'appropriation de cette séance, est abordé à partir de la diversité des écosystèmes paraliques; peu comparables à partir des connaissances antérieures, ces milieux vont être rassemblés à travers ce concept nouveau, qui s'avère ainsi créateur d'unité et de régularités. En effet, les représentations antérieures distinguaient des zones de mélange des eaux marines avec les eaux d'origine continentale : les eaux saumâtres, des interfaces soumises alternativement à l'émersion puis à l'immersion par des eaux marines: les surfaces de balancement des marées souvent appelées "estrans ", et des espaces rattachés au domaine continental mais pouvant être alimentés par la mer : les milieux évaporitiques (Guelorget et Perthuisot, 1983; Perthuisot et Guelorget, 1992). Le concept rassemble ces différentes classes en leur donnant une structure et une organisation homogènes dont l'exemple type est la lagune (Reynaud et al., 1995b).

\subsection{Situations-problèmes}

8 Une situation-problème doit permettre à chacun, en dix minutes, de faire individuellement le point de ces connaissances sur ces milieux à partir d'un problème concret. 
Question posée à l'expert :

Où prélever, dans la lagune de Guemsah, l'eau la mieux adaptée à l'alimentation d'une station d'aquaculture intensive de poissons (recherche de l'eau la moins chargée possible en matière organique)?

Données de départ :

- Localisation de la lagune de Guemsah : Mer Rouge, Égypte, latitude : $27^{\circ} 5 \mathrm{~N}$ (voir carte).

- La lagune est en communication avec la mer par une passe principale située au sud/sudouest (voir carte).

- Organisation biologique qualitative visible dans la passe :

1. d'un côté (indiqué sur la carte : 1 ) : nombreuses espèces marines (oursins, astérides, ...)

2. de l'autre côté (indiqué sur la carte : 2) : tapis cyanobactérien, peu d'espèces

- Présence d'un haut fond limitant la lagune au Sud - Direction des vents dominants connue (N-NE)

- La disponibilité des autres moyens nécessaires à l'installation de la station ne pose pas de problème.

\section{Exercice}

(sur la feuille jointe)

Indiquer par une surface hachurée en rouge sur la carte, la zone dans laquelle vous proposeriez d'installer la prise d'eau dans la lagune.

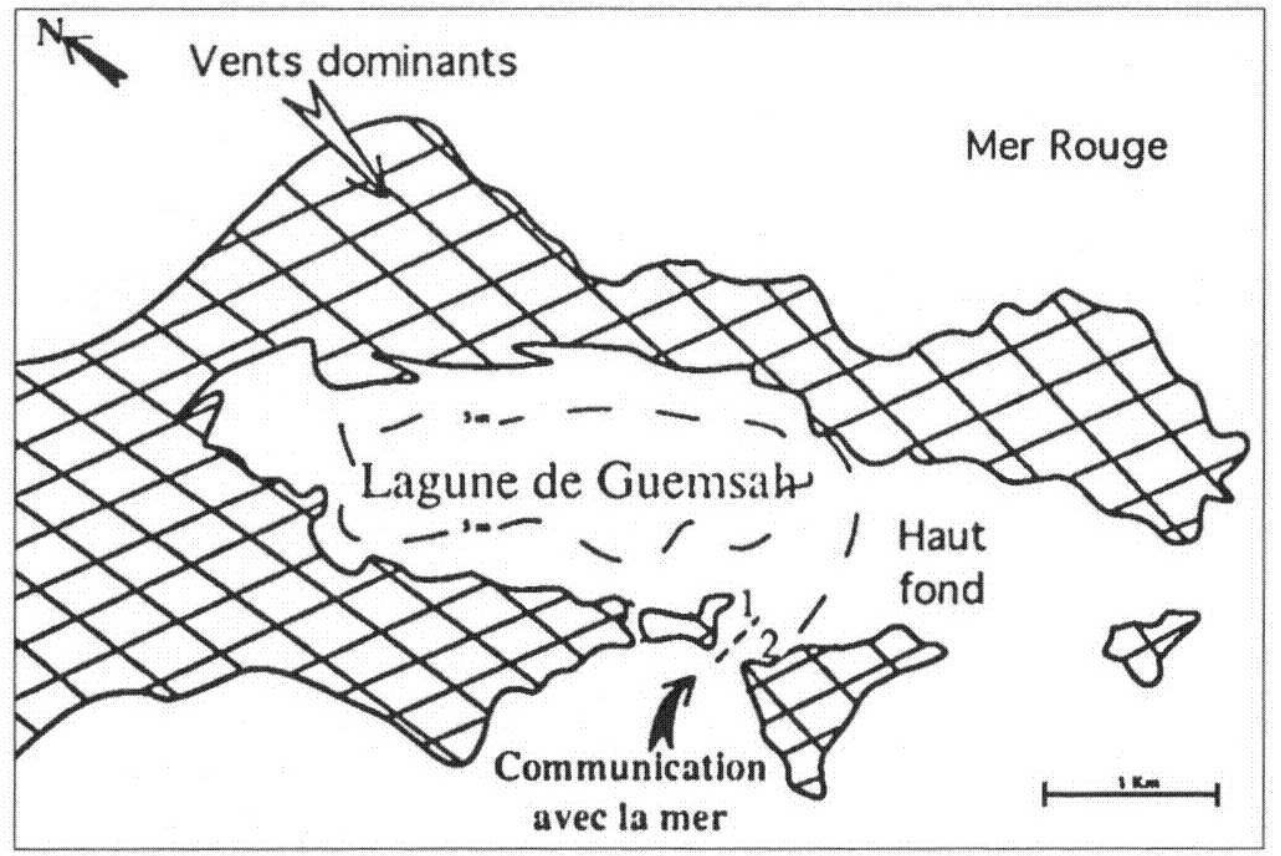

La première situation proposée est celle de la lagune de Guemsah. Un document rappelant la question posée à l'expert, l'énoncé de quelques données rapidement accessibles sur le terrain, et comportant un fond de carte est accompagné d'un agrandissement de la carte sur une seconde feuille permettant de formuler une réponse. Ce premier exercice a aussi l'avantage d'encourager la mobilisation de conceptions préalables (Giordan et De Vecchi, 1987). Chacun a ainsi la possibilité d'estimer leur efficience en matière de résolution de problème.

L'exposé qui suit consiste à présenter, en une vingtaine de minutes, des exemples caractéristiques des milieux pris en considération. 


\subsection{Premier exposé : quelques exemples}

11 Après avoir rappelé que les zones entre mer et continent ont longtemps été considérées comme des lieux de transition présentant un mélange des caractères des deux espaces, l'exemple de la lagune (stricto sensu) est représenté par le complexe palavasien ${ }^{3}$ (Guelorget et Perthuisot, 1983, fig. 26). Une définition synthétique de la lagune est citée à cette occasion (Reynaud et al., 1993). Dans ces étangs, des mesures de biomasse et de production des populations benthiques et phyto-planctoniques permettent de mettre en évidence une variation continue de ces paramètres en fonction de l'éloignement de la communication avec la mer. Le long de cet axe se superposent les principales communautés identifiées clans le milieu (Guelorget et Michel, 1979; Guelorget et Perthuisot, 1983). Pour décrire cette organisation, l'écosystème peut être subdivisé en six zones pouvant être distinguées à partir des êtres vivants qui les caractérisent (Guelorget et Perthuisot, 1992, fig. 9B).

Une zonation biologique similaire a été ainsi identifiée dans d'autres lagunes du pourtour méditerranéen (Guelorget et Perthuisot, 1983; 1992). La présence de certaines espèces dans les étangs palavasiens, présentant des salinités inférieures à la mer, comme dans des lagunes sursalées (Bahiret El Biban en Tunisie, par exemple), permet d'identifier des communautés pouvant servir à caractériser ces différentes zones indépendamment du gradient de salinité (Ibid.). Si cette distribution ne peut pas être corrélée à un gradient de salinité, il semble par contre possible de la mettre en relation avec l'intensité de l'influence de la mer, gradient désigné par le terme de "confinement» (Ibid.). La répartition de ces associations spécifiques dans une lagune schématique est présentée sur une figure faisant apparaître la zonation avec deux pôles extrêmes selon le signe du gradient de salinité du milieu par rapport à la mer (Guelorget et Perthuisot, 1992, fig. 9A). Cette figure est distribuée aux participants sur un polycopié qu'ils peuvent conserver jusqu'à la fin de la séance.

13 Sur cette base, la zone 1 est considérée comme la continuation du domaine marin ; c'est à la limite de cette zone que l'on passe au domaine paralique (Guelorget et Perthuisot, 1983 ; 1992). La zone 2 se caractérise par la disparition des espèces strictement marines (souvent sténohalines: qui ne supportent pas les variations de salinité, par opposition aux euryhalines). L'oursin, Paracentrotus lividus est une espèce caractéristique des zones 2 (Ibid.). La disparition des échinodermes permet de caractériser le passage dans la zone 3, et la palourde, Ruditapes decusatus en est caractéristique. Un annélide polychète, Glycera convoluta, moins connu mais assez répandu, l'est aussi. C'est aussi dans cette zone que l'on rencontre les densités d'huîtres plates, Ostreaedulis, les plus importantes (Ibid.). La zone 4 correspond à la présence d'espèces strictement paraliques, par exemple pour la faune la coque Cerastoderma glaucum, et pour la flore Ruppia spiralis (Ibid.). La zone 5 s'identifie à l'apparition de nombreux détritivores tel Sphœroma hookeri (crustacé isopode ressemblant au cloporte) et de gastéropodes brouteurs tels Hydrobia acuta. (Ibid.). Deux autres zones sont distingtuées, mais désignées comme zone 6 car elles correspondent au passage à une véritable zone de transition soit vers l'évaporitique soit vers le dulçaquicole, mais dans les deux cas leurs substrats sont colonisés par des tapis cyanobactériens (Ibid.).

14 Le même type de profil peut être obtenu à partir de l'étude de formation de type "bahira», dépressions d'origines continentales envahies par la mer lors de la transgression holocène (Perthuisot et Guelorget, 1992). C'est le cas de l'étang de Thau 
dans notre région, ou du lac Melah en Algérie. Ces formations se distinguent des lagunes (stricto sensu) essentiellement par leurs profondeurs supérieures (Ibid., fig. 4B).

La répartition par zones biologiques est cependant une représentation statique qui pourrait masquer la dynamique de ces milieux. L'organisation des milieux paraliques semble en effet pouvoir être conélée au fonctionnement hydrologique de ces milieux (Perthuisot et Guelorget, 1992; Guelorget et Perthuisot, 1983; 1992). L'étude de la circulation des eaux dans le lac Melah permet ainsi de mettre en corrélation le fonctionnement hydrologique et la répartition des êtres vivants dans ce milieu, mais fait ainsi apparaître une zone particulière où les eaux ayant séjourné dans le bassin forment un « ombilic » qui abrite de nombreux détritivores (Perthuisot et Guelorget, 1992, fig. 9).

la communication avec la mer est beaucoup plus large, comme clans le cas des estuaires, il existe généralement un «bouchon vaseux » - mobile en fonction de la marée et du débit du fleuve mais il ne semble pas possible d'identifier un seuil stable limitant le fond du bassin (Ibid.). Ainsi, la bathymétrie ne permet pas de distinguer les limites du Limski Kanal du côté de la mer (Ibid., fig. 1).

17 La possibilité de reconnaître une zonation biologique devient beaucoup plus évidente (et plus connue) sur les zones de balancement des marées (zones d'estrans) pour lesquelles la variation continue de l'influence de la mer, le confinement ne s'exprime plus seulement selon un axe horizontal. Cette possibilité s'applique aussi aux types intermédiaires. L'organisation d'El Kantara est par exemple typique d'une formation «bahira - estran » (Ibid. 1992, fig. 6).

18 L'application du schéma de l'organisation biologique des écosystèmes paraliques à différentes échelles permet d'exprimer les variations dans la composition des peuplements en terme de confinement et de rattacher ainsi de grands bassins au domaine paralique.

19 La mer Caspienne est un bassin complètement fermé, séparé de la mer mais présentant des teneurs en sels qui témoignent de l'existence d'une communication clans le passé (Guelorget et Perthuisot, 1983, fig. 44). Les données biologiques, et notamment la présence d'espèces typiques des milieux paraliques, permettent de retrouver l'organisation caractéristique de ces milieux (Ibid., fig. 47). L'absence des zones I et II s'explique par l'ancienneté de la séparation avec la mer, mais ne permet pas de considérer ce milieu comme un écosystème paralique stricto sensu. L'exemple reste cependant intéressant car il représente de manière caricaturale l'évolution de ces milieux dont la dynamique à long terme conduit vers la continentalisation. La mer Baltique peut, par contre, être considérée comme un véritable milieu paralique. Elle présente alors une modalité particulière d'expression $d u$ confinement lorsque certaines zones profondes sont relativement isolées des mouvements d'eau de surface : il est alors possible de parler d'un confinement bathymétrique (ibid., fig. 65). Dans ces zones extrêmes en terme de confinement les sédiments sont, encore une fois, caractérisés par une grande richesse en matière organique. Si la matière organique semble être une composante importante des sédiments dans les milieux paraliques, d'où peut-elle provenir?

L'activité biologique, très importante dans les écosystèmes paraliques, pourrait en être la source principale puisque les milieux paraliques peuvent être caractérisés par une production biologique bien supérieure à celle des autres écosystèmes de la biosphère (Ibid., fig. 34). 
21 Cette productivité considérable semble même pouvoir être corrélée aux ressources démersales : le niveau de capture de poissons et crustacés dans les zones littorales de la Méditerranée peut être mis en relation avec la proximité des milieux paraliques (Ibid., fig. 36). Ainsi les zones côtières les plus riches bordent les côtes abritant des milieux paraliques importants et productifs. En revanche, les régions caractérisées par une faible productivité des pêches se distinguent aussi par l'absence de lagune côtière (Amanieu et Lassere, 1981).

Cette richesse biologique des régions abritant des milieux paraliques semble pouvoir expliquer l'intérêt que leur ont porté les populations humaines au cours de l'histoire et leur mise en exploitation depuis très longtemps. Aujourd'hui, la compréhension de leur mode de fonctionnement permet d'envisager la gestion de leur productivité et peut ainsi s'appliquer au développement de l'aquaculture dans les écosystèmes paraliques: les différentes formes d'exploitation du milieu peuvent être aménagées en fonction des contraintes particulières de chacune (Dutrieux et Guelorget, 1988, fig. 6).

\subsection{Réflexion : les points communs des milieux présentés}

23 À la suite de cet exposé, les participants disposent de cinq minutes de réflexion pour rechercher, chacun pour soi, les points communs, les caractéristiques communes à tous les milieux présentés. Le produit de ces réflexions est ensuite proposé à l'ensemble du groupe tandis que l'animateur note toutes les propositions sur un tableau. L'animateur veille cependant à faire expliciter certaines suggestions lorsqu'il juge qu'elles pourraient être reformulées sous forme de plusieurs alternatives ${ }^{4}$. Mais il semble préférable que toutes les propositions des participants soient prises en compte, même si celles-ci s'avèrent redondantes. Les occurrences faisant l'objet d'une discussion peuvent cependant être distinguées (en les soulignant par exemple). Bien que ce travail soit proposé à la fin de l'exposé, alors que Barth $(1987,1993)$ préconise une démarche simultanée, cet exercice reste relativement proche de la méthode proposée par cet auteur pour distinguer les attributs essentiels d'un concept.

24 Lorsque les participants ont énoncé toutes les caractéristiques qu'ils ont pu discerner, l'animateur présente les différents types d'attributs qui peuvent être distingués (Reynaud et al., 1995a).

\subsection{Deuxième exposé : les attributs du concept}

25 À ce stade, un deuxième exposé d'une vingtaine de minutes est proposé afin de commenter les attributs du concept qui ont été pris en compte pour en donner une formalisation sous forme d'un schéma (qui est distribué) ${ }^{5}$.

\subsubsection{Premier attribut essentiel : milieu aquatique éventuellement temporaire}




\subsubsection{Deuxième attribut essentiel : en communication puis ou moins large et/ou périodique avec la mer} matériaux sédimentaires (d'origine minérale et/ou organique); ce sont des réceptacles toujours bien délimités par des barrières physiques (au sens large) : géologique (ex. : un lido ou barrière sédimentaire), physique (ex. : thermocline) ou biologique (ex. : herbier). Cela implique que l'on peut toujours tracer les limites d'un domaine paralique. La qualité des matériaux sédimentaires dépend toutefois de trois facteurs: l'influence de la mer, l'influence du continent et l'activité du milieu lui-même (produisant des carbonates et la matière organique). Mais l'intense production biologique de l'écosystème donne lieu à la formation de sédiments surtout biogéniques.

\subsubsection{Quatrième attribut essentiel : milieu à forte production biologique}

31 Une grande productivité des premiers niveaux trophiques (production bactérienne et primaire) est la base de cette forte production biologique. Comparativement, la production bactérienne peut être estimée entre 1 et $10^{4} \mathrm{mg} \cdot \mathrm{m}^{-3} \cdot \mathrm{j}^{-1}$ de carbone dans les milieux lagunaires, la production algale peut varier d'environ 50 à $5000 \mathrm{mg} \cdot \mathrm{m}^{-3} \cdot \mathrm{j}^{-1} \mathrm{de}$ carbone. La prolifération rapide des autotrophes (algues et cyanobactéries) et des décomposeurs (bactéries) en réponse aux variations de milieu (eutrophisation par exemple) témoigne ainsi d'une capacité de production très importante. Notons cependant que les milieux paraliques se caractérisent souvent par une faible richesse spécifique compensée par une densité élevée des individus.

\subsubsection{Cinquième attribut essentiel : milieu structuré et organisé sur le modèle du confinement}

La distribution des espèces dans le milieu paralique s'organise schématiquement selon la distance à la mer. Cette organisation est une expression du confinement, rendant compte de l'intensité de l'influence marine. Il est possible d'estimer une valeur globale du confinement pour un bassin paralique par le temps nécessaire au renouvellement complet des eaux paraliques. Mais, pour estimer sa valeur en un point donné du bassin, on peut utiliser une échelle qualitative se référant à la distribution des espèces de la macrofaune benthiques dans le milieu. La zonation biologique des espèces benthiques (macrofaune) permet ainsi de définir 6 degrés (ou zones) de confinement en ce qui concerne la portion du domaine paralique proche de la mer, mais elle ne rend pas compte

Tréma, 9-10 | 2010 
des gradients de salinité. Il faut cependant noter que la plus grande partie de chaque écosystème paralique s'étend sur un nombre restreint de zones de confinement.

\subsubsection{Sixième attribut essentiel : écosystème en dynamique continue} contrôlée essentiellement par l'hydrodynamique : les courants existants dans les bassins paraliques influencent la répartition des divers organismes peuplant ces milieux. Ces courants pouvant être régis par divers facteurs (tels la forme du bassin, le sens et l'intensité de la communication avec la mer, le vent dominant, les différences de densité de la masse d'eau, ...), ils sont souvent très variables, et les divers gradients existant dans le milieu subissent les conséquences de cette variabilité. Cependant, l'activité des autotrophes et des décomposeurs permet d'assurer l'équilibre écologique du milieu. En effet, on considère généralement que les crises dystrophiques (malaïgues), touchant les zones les plus confinées et entraînant une minéralisation massive de la matière organique excédentaire, rétablissent rapidement les conditions d'équilibre. Ce type de régulation engendre cependant une dynamique évolutive à long terme: étant des lieux de sédimentation rapide et importante, les écosystèmes paraliques sont appelés à évoluer, souvent dans le sens d'un comblement.

Il importe de souligner que ces six attributs doivent être reliés par la conjonction "et » pour rappeler qu'ils sont tous nécessaires et que l'ensemblesuffit à la définition du concept. Si, pour un milieu donné il manque un attribut, l'écosystème ne pourra pas être qualifié de paralique (selon cette définition).

\subsection{Résolution collective du problème de Guemsah}

En reprenant le problème proposé en début de séance l'animateur montre comment la formalisation des attributs permet d'avancer une réponse. Ainsi, la lagune de Guemsah est un milieu aquatique (attribut $N^{\circ} 1$ ), bassin bien délimité (attribut $N^{\circ} 3$ ). L'organisation des espèces (attribut $N^{\circ}$ ) visible sur les côtés de la passe permet de penser qu'une entrée d'eaux marines (attribut $N^{\circ}$ )se fait par un côté de la passe (celui où l'on trouve les espèces d'origine marine), l'autre côté permettant l'évacuation des eaux ayant séjourné dans la lagune. Le sens de circulation des courants étant ainsi inféré (attribut $N^{\circ} 6$ ), l'eau nécessaire à la station d'aquaculture peut être prélevée à l'entrée des courants où elle ne sera pas chargée de la matière organique produite dans le milieu (attribut $N^{\circ} 4$ ).

\subsection{Débat socio-cognitif autour de la situation-problème de l'étang du Prévost}

Partant des suggestions de Doise et Mugny (1981), les phases de débat sont mises en place afin de développer une communication au sein du groupe ( interaction sociale») favorable à l'élaboration de nouvelles connaissances ("restructuration cognitive »); ce que ces auteurs nomment « le conflit sociocognitif ». Pour introduire la démarche qui est utilisée, l'animateur aborde rapidement des exemples historiques de notions scientifiques ayant fait l'objet de longues controverses (la circulation sanguine de Harvey, le système héliocentrique de Copernic) en liant l'ampleur de la durée du débat à la difficulté que les chercheurs peuvent avoir à communiquer leurs points de vue respectifs et à s'enrichir de 
celui des autres. Il propose alors aux participants d'expérimenter, sur la base d'un problème qui leur est proposé, un débat avec trois règles pour catalyser l'évolution des représentations. Pour cela il demande que chacun tente, tout d'abord, de se forger une opinion personnelle sur la réponse à donner à ce problème.

Question posée à l'expert :

Où prélever l'eau la mieux adaptée à l'alimentation d'une station d'aquaculture intensive de poissons (Loups et Dorades) installée sur un lido?

Données de départ :

- Situation géographique : Méditerranée, France, latitude : $43^{\circ} 7 \mathrm{~N}$.

- Description du site : près de la ville de Palavas, sur le lido séparant l'étang du Prévost de la mer, a été installée une station d'aquaculture intensive expérimentale.

- L'eau prélevée devra alimenter des bassins de taille réduite dans lesquels la forte densité de poissons implique une consommation d'oxygène importante et engendre une production élevée de déchets organiques.

- Observation : La zone de l'étang en face de la station (A) présente des dépôts riches en matière organique. À l'opposé par rapport au grau (B) les substrats durs sont colonisés par des huîtres sauvages. À l'Est de la jetée (C) on trouve des sédiments grossiers et il est possible de recueillir des nombreuses espèces marines. De l'autre côté de la jetée (D) des dépôts sableux abritent des espèces détritivores ; une zone où se rencontrent des mollusques filtreurs est aussi nettement observable (voir carte).

Exercice (sur la feuille jointe) : Indiquer l'emplacement où vous proposeriez d'installer la prise d'eau par une des lettres indiquées sur la carte.

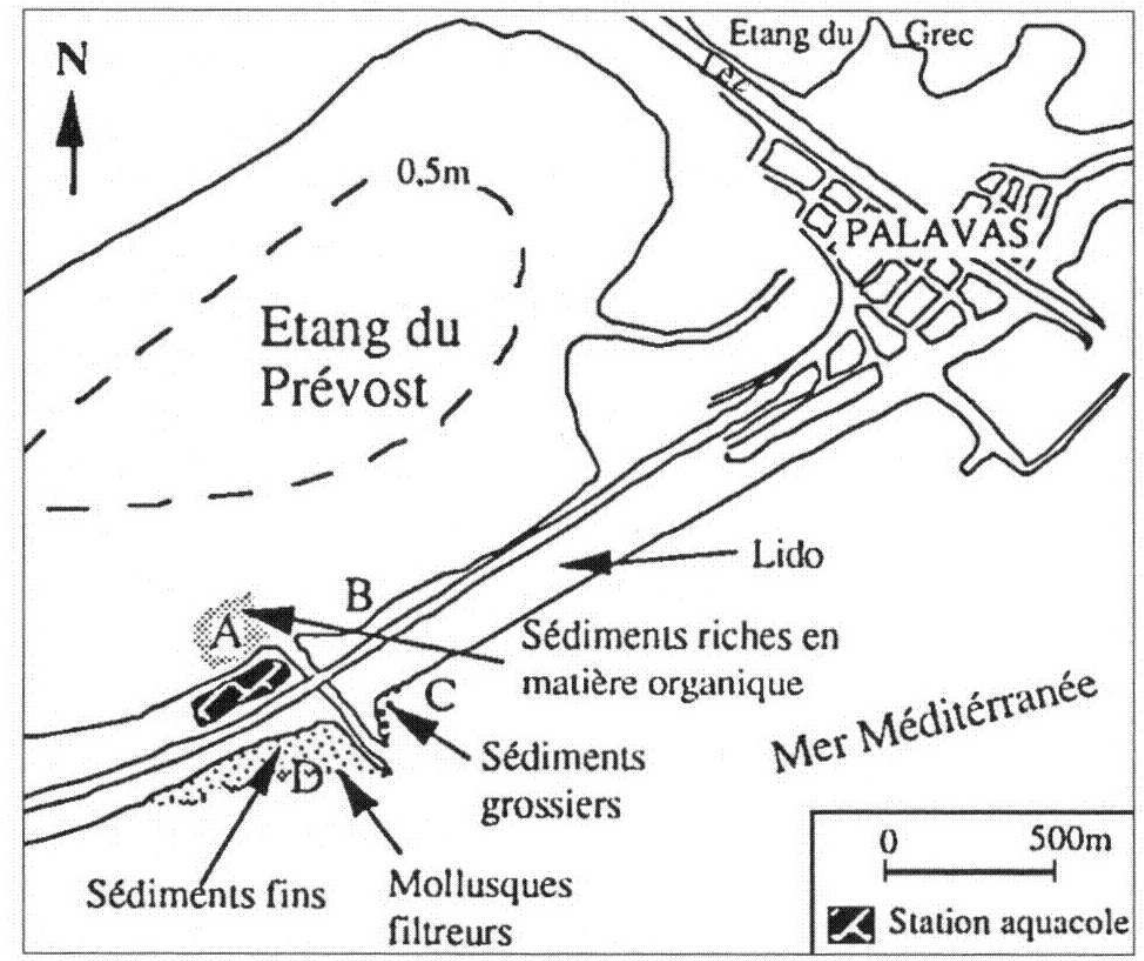

Pour satisfaire aux propositions de Doise et Mugny (Ibid.), les participants doivent exprimer des opinions divergentes. Nous avons donc pris soin de choisir un problème qui admette un nombre de solution limité et pour lequel nous avions testé au préalable les potentialités à induire des avis variés. Les participants sont donc invités à réfléchir sur la 
situation-problème de Palavas. Un premier sondage (dont les résultats sont collectés sur un tableau) permet après cela de se rendre compte de la diversité des avis en présence. L'animateur invite ensuite l'assistance à considérer que « chacun a de bonnes raisons de penser ce qu'il pense - "bonnes" signifiant "intrasubjectivement valables" » (postulat de cohérence constituant la première règle) et que ses arguments méritent d'être exposés à l'assistance (deuxième règle). Mais, pour être sûr que les arguments invoqués pour défendre une opinion sont bien compris par les « opposants ", une personne ayant un avis différent est incitée à reformuler le développement de la thèse adverse (troisième règle). La qualité de la reformulation est soumise à l'appréciation de l'énonciateur qui peut éventuellement repréciser les points n'ayant pas été repris ou choisir d'accorder quittus. Cette exigence de reformulation a pour objectif de signifier à l'opposant que ces arguments ont bien été assimilés, mais elle a aussi pour effet de permettre à chaque parti de s'approprier les arguments adverses. La première position qui est défendue est celle rassemblant le moins d'adeptes. Ceci se justifie car si les partisans de la solution la plus plébiscitée s'expriment les premiers, ils ont toutes les chances de rallier rapidement leurs opposants au nom d'un conformisme. Les minorités sont d'ailleurs encouragées par des commentaires tels que : «N'oubliez pas que Galilée était seul pour affirmer que la terre tourne autour du soleil! ».

Lorsque toutes les solutions ont été justifiées par leurs défenseurs et qu'aucun participant ne souhaite compléter l'une des argumentations, il est possible de refaire le point sur les positions respectives des participants car certains d'entre eux ont pu modifier leur opinion. Une position intermédiaire (appelée "floue ») peut même être proposée à ceux qui n'arrivent plus à choisir une solution qui leur donne satisfaction. Quand il existe des individus ayant modifié leur opinion de départ il est alors intéressant de leur demander d'expliciter les raisons de leur changement de position en insistant notamment pour qu'ils recherchent l'argument décisif dans cette rectification. Ceci permet en effet de susciter une réflexion sur le poids accordé à un argument particulier. À ce stade toutes les objections peuvent être avancées pour enrichir la discussion. L'animateur du débat peut cependant demander la reformulation d'un raisonnement par une tierce personne s'il la juge nécessaire pour sortir d'une impasse qui résulterait d'une « incommunication ». Le débat se termine lorsque les controverses ne sont plus alimentées par des discussions critiques; en théorie, cette situation se présente à l'instant où une des réponses proposées au problème est devenue évidente pour tout le monde. L'expérience montre qu'il faut prévoir plus d'une heure pour atteindre ce stade.

\subsection{Deux situations-problèmes pour faire le point (Karavasta et Monastir)}

39 L'objectif du dispositif étant l'acquisition de capacités à résoudre le type de question posée à des experts des milieux paraliques, les connaissances mobilisées au cours de la séance doivent pouvoir être maîtrisées dans des contextes différents de ceux déjà abordés. Pour terminer la séquence, deux autres situations-problèmes sont donc proposées aux participants afin qu'ils puissent mettre à l'épreuve les capacités qu'ils ont pu acquérir grace au dispositif mis en œuvre. Celle de Karavasta, présentée ci-desous, peut sembler assez simple puisqu'il s'agit d'une lagune typique. Il faut pourtant remarquer le bassin en formation à l'avant de la lagune actuelle. 
Question posée à l'expert :

Où pourrait-on installer des tables d'élevage conchylicoles (huîtres) dans la région de Karavasta pour obtenir une production optimale?

Données de départ :

- Situation géographique : Mer Adriatique, Albanie, $41^{\circ} \mathrm{N}$.

- Observation :

1. dans l'enceinte de la lagune on peut pêcher, en grande quantité, des mulets et athérines, espèces qui affectionnent les zones riches en micro-organismes détritivores ;

2. on ne récolte des oursins qu'à quelques kilomètres au large de la lagune actuelle.

- Les coquillages que l'on souhaite exploiter:

1. à l'état sauvage, s'installent en eaux peu agitées, mais bien oxygénées ;

2. sont des animaux filtreurs (ils se nourrissent de matière organique en suspension).

Exercice (sur la carte jointe) : Indiquer par une zone hachurée en rouge sur la carte l'emplacement où vous proposeriez d'installer les tables d'élevage (surface d'environ $1 \mathrm{~km}^{2}$ ).

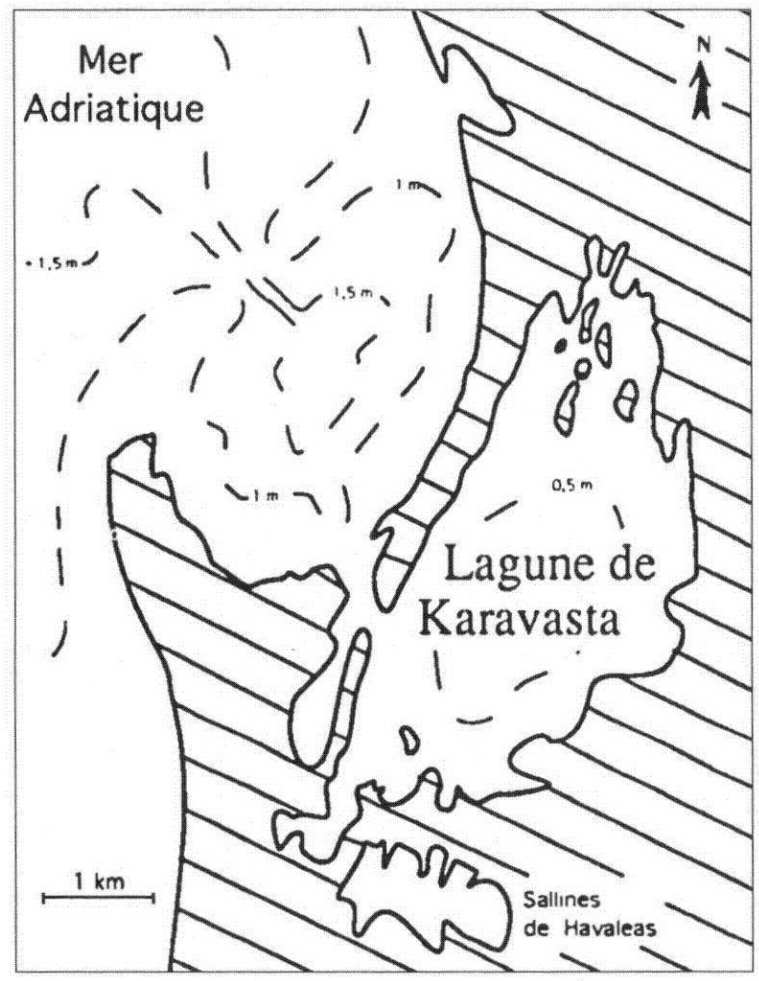

Ces situations-problèmes permettent aussi d'évaluer les effets de notre dispositif.

\section{Synthèse des résultats obtenus dans l'enseignement}

Si l'objectif de l'enseignement est d'acquérir une capacité à réinvestir des connaissances dans la résolution de "problèmes non routiniers ", les étudiants devraient être capables de proposer une solution à des problèmes simplifiés à partir de rapports d'expertises. Nous avons donc entrepris une évaluation de la capacité à réinvestir des connaissances dans des situations problèmes chez des étudiants de l'université de niveau bac +3 ayant participé à des séquences d'enseignement sur le thème qui nous intéresse. Les résultats 
que nous avons obtenus à partir des réponses données aux situations problèmes montrent que :

- sur un groupe d'étudiants (G0) ayant suivi un enseignement « traditionnel » de 12 heures sur le thème, puis une séquence de 2 heures d'exposé magistral pour présenter notre modèle, $31 \%$ donnent la réponse attendue à la situation de Karavasta et $3 \%$ pour Monastir ;

- sur un groupe d'étudiant (GF) ayant participé à la séquence expérimentale de 3 heures que nous venons de décrire, $40 \%$ expriment la réponse attendue à la première situation et $70 \%$ pour la deuxième.

42 Les réponses données par chaque groupe sont indiquéessur la figure 1 ainsi que les résultats que nous avons obtenus progressivement, avec des groupes intermédiaires nous ayant permis de perfectionner notre dispositif didactique.

Ces résultats doivent cependant être pris avec prudence car de nouveaux perfectionnements peuvent être amenés au dispositif afin de confirmer nos interprétations. En effet, nous pensons introduire en fin de séance une situationproblème concernant un milieu qui ressemblerait à un écosystème paralique mais qui n'en serait pas un selon notre définition (i.e. si il manque un ou plusieurs attributs).

Il semble enfin important de souligner que les groupes que nous avons évalués sont constitués d'étudiants ayant une formation scientifique : ceci nous a d'une part dispensé d'approfondir des notions fondamentales telles que celle de "productivité biologique ", et d'autre part, les étudiants ont bénéficié d'une formation préalable aux pratiques de récolte et de reconnaissance d'espèces, acquis qui pourraient s'avérer indispensable à l'acquisition du concept.

\section{4. les éléments transférables de cette approche}

Ce type d'approche pourrait-il être utilisé pour d'autres concepts de biologie? pour des concepts d'autres disciplines?

Avant d'aborder ces questions il faut préciser une limite fondamentale de notre approche: nous pensons que l'objectif de l'enseignement doit être de développer une capacité à résoudre des problèmes qui peuvent se poser en dehors de la situation d'enseignement elle-même et pour lesquels la solution ne peut pas être obtenue par la simple application d'un algorithme de résolution (ce que nous avons appelé les « problèmes non routiniers »). 
Fig. 1 : Proportion des réponses attendues (par rapport au total des réponses) en fonction des étapes de la formation et du développement du dispositif.

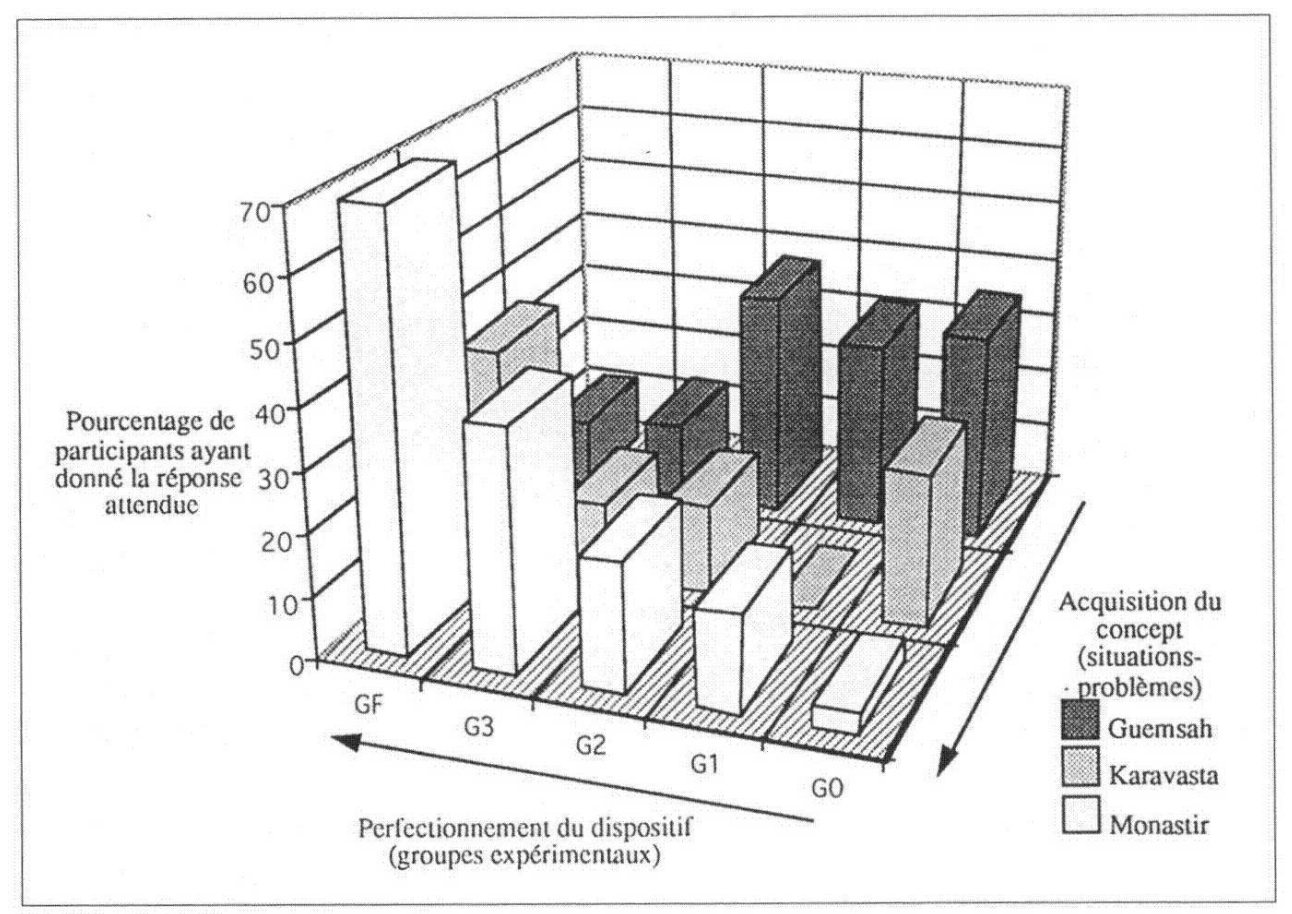

Certains éléments de notre approche semblent alors transférables :

1. Rechercher, identifier les notions conceptuelles dont on vise l'acquisition pour un public donné : il s'agit de reconnaitre les outils théoriques qui permettent d'appréhender une classe de problèmes données (Reynaud et al., 1993).

2. Formaliser le concept identifié, éventuellement avec le concours des chercheurs spécialisés, pour en dégager les attributs essentiels et conséquents (Reynaud et al., 1995a).

3. Adapter et proposer des situations problèmes dont la résolution nécessite l'utilisation du concept à travers ses attributs (Ibid.).

4. Créer des "débat sociocognitif» susceptibles d'engendrer des conflits cognitifs intrasubjectifs à l'aide des situations problèmes. La dynamique de telles séquences fait fond sur des motivations intrinsèques qui favorisent tine véritable appropriation des concepts (Favre et Favre ; 1993, 1996).

\section{Conclusion}

L'écologie semble nous donner un exemple particulier de liaison directe entre les connaissances issues de la recherche et la résolution de problèmes pratiques. L'émergence d'un concept répond alors à l'identification d'une classe de problèmes particuliers. Lorsqu'il s'agit d'enseigner, l'existence de cette liaison permet de légitimer une pédagogie s'appuyant sur la résolution de problèmes pratiques (Pochet, 1995). La problématique de l'enseignement de la biologie est-elle vraiment différente?

L'objectif de cet enseignement ne pourrait-il pas être le développement de capacités d'expert du type: reconnaître et comprendre le fonctionnement d'un système biologique? Certes, le savoir mis en jeu dans les enseignements de biologie traditionnels constitue une structure permettant de développer ces capacités de traitement de 
l'information, mais il ne les fonde pas. C'est un outil, avec ses spécificités, mais son maniement ne va pas de soi.

Avec le dispositif que nous proposons, un travail qui reste théorique mais appliqué à des situations réelles semble donner aux apprenants la capacité à mobiliser des concepts scientifiques pour résoudre des problèmes d'aménagement des milieux littoraux. Une telle approche pourrait placer les apprenants en situation de construction de concepts par une activité de transformation réelle de leurs conceptions. En favorisant l'intervention de tous les participants et en autorisant l'innovation, elle devrait permettre d'acquérir des compétences sociales indispensables à l'épanouissement des citoyens.

Dans cette perspective démocratique, les objectifs d'ouverture au changement de conception permettraient d'offrir à chaque individu les moyens de participer à de réels débats sur le type de développement scientifique et technique souhaité et donc d'être acteur de l'évolution de notre société.

\section{BIBLIOGRAPHIE}

AMANIEU M. \& LASSERE G., « Niveaux de production des lagunes littorales méditerranéennes et contribution des lagunes à l'enrichissement des pêches démersales ", in Études et Revues, $\mathrm{N}^{\circ} 58$, C.G.P.M., 1981, p. 81-94.

BARTH B.M., L'apprentissage de l'abstraction, Paris, Retz, 208 p., 1987.

BARTH, B.M., Le savoir en construction, Paris, Retz, 208 p., 1993.

BROUSSEAU G., Quelques concepts fondamentaux en didactique des mathématiques, E.P.S. Contenus et didactique, 1986, p. 269-277.

DELÉAGE J.-P., Histoire de l'écologie, Paris, La Découverte, 330 p., 1991.

DOISE W. \& MUGNY G., Le développement social de l'intellignence, Paris, InterEditions, 199 p., 1981.

DROUIN J.-M., L'écologie et son histoire, Paris, Flammarion, 218 p., 1991, (réédition 1993).

DUTRIEUX E. \& GUELORGET O., "Ecological planning : A possible method for the choice of aquacultural sites", in Ocean \& Shoreline Management, $\mathrm{N}^{\circ} 11,1988$, p. 427-447.

FAVRE D. \& FAVRE C., « Un modèle complexe des motivations humaines », in Revue de Psychologie de la Motivation, $\mathrm{N}^{\circ} 16,1993$, p. 27-42.

FAVRE D. \& FAVRE C., « Crise et apprentissage : quelles motivations ?, » in Cahiers Pédagogiques. Hors Série « Motivation », 1996, p. 41-49.

GIORDAN A. \& DE VECCHI G., Les origines du savoir : des conceptions des apprenants aux concepts scientifiques, Paris, Delachaux et Niestlé, 214 p., 1987.

GUELORGET O. \& PERTHUISOT J.-P., Le domaine paralique - Expressions géologique, biologiques et économiques du confinement, Paris : 16ªrr., Presse École Normale Supérieure, 136 p., 1983. 
GUELORGET O. \& PERTHUISOT J.-P., "Paralic ecosystems - Biological organization and functionning”, in Vie Milieu, Vol. 42 : N² 2, 1992, p. 215-251.

GUELORGET O. \&MICHEL P., « Les peuplements benthiques d'un étang littoral languedocien l'étang du Prévost (Hérault) », in Téthys, Vol. 9 : Nº 1, 1979, p. 49-77.

PERTHUISOT J.-P. \& GUELORGET O., « Morphologie, organisation hydrologique, hydrochimie et sédimentologie des bassins paraliques », in Vie Milieu, Vol. 42 : N² 2, 1992, p. 93-109.

POCHET B., « Le "Problem-Based Learning" une révolution ou un progrès attendu ? ", in Revue Française de Pédagogie, $\mathrm{N}^{\circ} 111,1995$, p. 95-107.

REYNAUD C., GUELORGET O., RIEUCAU J. \& FAVRE D., « Approche didactique des représentations du concept de Lagune », in Actes du colloque «Okéanos 93 : Le système littoral méditerranéen, connaissance, gestion, protection », Montpellier, 1993, p. 214-216.

REYNAUD C., GUELORGET O. \& FAVRE D., "Didactic approach of the paralic ecosysytem concept", in Actes du colloque "Baltic sea and Mediterranean sea - A comparative ecological approach of coastal environments and paralic ecosystems", Montpellier, 1995a, p. 14-26.

REYNAUD C., GUELORGET O. \& FAVRE, D., « Contribution didactique à la modélisation du concept de milieu paralique et application à l'étude des conceptions des élus locaux ", injournal de Recherche Océanographique, Vol. 20 : N 1-2, (sous presse), 1995b.

\section{NOTES}

1. Le qualificatif de "paralique" devra être considéré comme le véhicule de l'information relative au concept étudié, nous reprendrons, ensuite, les vocables utilisés dans les publications spécialisées en utilisant donc les termes de "milieux ", " écosystèmes ", " domaines ", « environnements », et « bassins » paraliques.

2. Par opposition aux attributs non essentiels qui peuvent caractériser un exemple particulier mais qui ne sont pas représentatifs de tous les exemples du concept.

3. Ce document est présenté sur transparent pendant la séance. De même que tous ceux qui ont déjà été publiés, il n'est pas reproduit ici mais les références en italique permettent de le retrouver.

4. «Bassin relativement isolé du domaine marin » peut être explicité et devenir par exemple « zone bien délimitée » et « milieu en relation avec le domaine marin ».

5. Pour retrouver ce schéma et les références aux travaux d'écologie qui ont servis de base à cette formalisation, voir Reynaud et al., 1995a.

\section{RÉSUMÉS}

L'objectif de l'atelier était de proposer une séquence d'enseignement utilisant un dispositif de présentation des caractéristiques du concept d'écosystèmes paraliques et visant à développer des compétences pour résoudre des "problèmes non routiniers" concernant les écosystèmes littoraux. Outre la description du dispositif didactique expérimental construit à cet effet, une 
synthèse des résultats préalablement obtenus avec des étudiants en licence de biologie est présentée.

The goal of the workshop was to propose a sequence of classroom sessions built around the presentation of the characteristics of the concept of wetland ecosystem, and aiming at developing skills in solving "non routine problems" concerning coastal ecosystems. Besides the description of the especially devised experimental didactic setup, the article presents a synthesis of the preliminary results obtained with "licence" level biology students.

INDEX

Keywords : problem situation, socio - cognitive debate, wetland ecosystem

Mots-clés : débat socio - cognitif, écosystème paralique, situation - problème

\section{AUTEURS}

\section{CHRISTIAN REYNAUD}

Laboratoire de modélisation de la relation pédagogique, équipe ERES, université de Montpellier II DANIEL FAVRE

Laboratoire de modélisation de la relation pédagogique, équipe ERES, université de Montpellier II 